\title{
Regulatory Traits: Cultural Influences on Cultural Evolution
}

\author{
Alberto Acerbi, Stefano Ghirlanda, and Magnus Enquist
}

\begin{abstract}
We use the term regulatory traits to indicate traits that both regulate cultural transmission (e.g., from whom to learn) and are themselves culturally transmitted. In the first part of this contribution we study the dynamics of some of these traits through simple mathematical models. In particular, we consider the cultural evolution of traits that determine the propensity to copy others, the ability to influence others, the number of individuals from whom one may copy, and the number of individuals one tries to influence. We then show how to extend these simple models to address more complex human cultural phenomena, such as ingroup biases, the emergence of open or conservative societies, and of cyclical, fashion-like, increases and decreases of popularity of cultural traits. We finally discuss how the ubiquity of regulatory traits in cultural evolution impacts on the analogy between genetic and cultural evolution and therefore on the possibility of using models inspired by evolutionary biology to study human cultural dynamics.
\end{abstract}

\footnotetext{
A. Acerbi $(\square)$

Centre for the study of cultural evolution, Stockholm University, Stockholm, Sweden e-mail: alberto.acerbi@gmail.com

S. Ghirlanda

Department of Psychology, Brooklyn College, New York, NY, USA

Centre for the study of cultural evolution, Stockholm University, Stockholm, Sweden e-mail:drghirlanda@gmail.com

M. Enquist

Centre for the study of cultural evolution and Department of Zoology, Stockholm University, Stockholm, Sweden

e-mail: magnus.enquist@intercult.su.se
} 


\section{Introduction}

We use the term regulatory traits to indicate cultural traits that both regulate cultural transmission (e.g., from whom and when to learn) and are themselves subject of cultural transmission. In modern western societies, for example, parents actively transmit the idea that children should learn from schoolteachers, or teenagers attempt to persuade their peers not to listen to adults. Depending on our experiences, we can learn to be conformist or anti-conformist, which, in turn, can modify the outcome of future social interactions. Many other examples are possible.

Regulatory traits constitute, in our view, an important difference between cultural and genetic evolution. Tools from evolutionary biology have been extensively used to develop cultural evolutionary theory $[1,2]$, based on the assumption that the process of cultural change shares some fundamental properties with the process of genetic change: namely, variation, inheritance, and competition [3,4]. While most researchers agree that culture has these properties, many have questioned whether they are sufficient to consider genetic and cultural evolution as essentially similar [5-7]. Some differences are obvious: for example, whereas genetic transmission is necessarily from parents to offspring, cultural transmission can, in principle, occur between any two individuals [8]. Thus models of genetic evolution certainly need to be modified to apply them to cultural evolution. The main question, however, is whether cultural and genetic evolution are different enough that it is misleading to study them using essentially the same models.

Population genetics models generally assume that the rules of transmission are stable. Consequently the routine assumption of cultural evolution models inspired by evolutionary biology is also that the rules of transmission are stable, or change slowly under genetic influence $[1,9]$. However, this is not necessarily true: regulatory traits seem ubiquitous in cultural evolution-and not in genetic evolution (see Sect.4). Here we explore the consequences of this difference on cultural dynamics.

We first use simple, yet very general, mathematical models to show that regulatory traits may have a profound impact on cultural evolution. Then we show, using a mix of mathematical models and computer simulations (see also [10-12]), how to extend these models to investigate real-world cultural phenomena such as in-group biases, conservatism, and fashion dynamics.

\section{Models}

Our basic assumption is that, in social interactions, individuals tend to copy each other's cultural traits. This tendency, however, is modulated by the traits themselves. For example, someone with the idea that people are trustworthy is expected to copy others more often than someone who mistrust people. In our models, we either calculate the expected effect of an interaction between two 
randomly chosen individuals, or the expected change, per time step, in the number or proportion of individuals carrying a particular trait. The key insight exploited in such calculations is that a regulatory trait also regulates its own transmission. Using the same example above, acquiring the idea that people are trustworthy will increase copying and thereby foster opportunities for modifying the same idea. Our calculations show clearly the dynamical forces generated by regulatory traits and enable us to predict the end-state of the population when only one trait is considered. When many regulatory traits influence cultural evolution, in-depth analysis of the dynamical equations and computer simulations are generally required and the resulting dynamics can be highly nontrivial, as we will show in Sect. 3.

\subsection{Openness}

We define openness, $p$, as the probability that an individual changes in a social interaction $\left(1-p\right.$ is thus a measure of conservatism). Let $p_{i}$ be the openness of individual $i, p_{i} \in[0,1]$, and let us consider a social interaction between individuals $i$ and $j$ such that $i$ is less open $j, p_{i}<p_{j}$. Let $E\left[\Delta p_{i}\right]$ be the expected change in $i$ 's openness caused by the interaction. Because individuals tend to copy each other, the interaction is expected to increase $p_{i}$ and decrease $p_{j}$ :

$$
E\left[\Delta p_{i}\right]>0 \quad E\left[\Delta p_{j}\right]<0
$$

Since $i$ is less open, she will on average change less than $j$, by hypothesis:

$$
\left|E\left[\Delta p_{i}\right]\right|<\left|E\left[\Delta p_{j}\right]\right|
$$

It follows that the average openness of the two interacting individuals, $\frac{1}{2}\left(p_{i}+p_{j}\right)$, is expected to decrease as a consequence of the interaction:

$$
E\left[\Delta \frac{1}{2}\left(p_{i}+p_{j}\right)\right]=\frac{1}{2} E\left[\Delta p_{i}\right]+\frac{1}{2} E\left[\Delta p_{j}\right]<0
$$

where the conclusion that the change is negative follows directly from Eqs. (1) and (2). Thus social interactions tend to decrease population openness, until all variation in openness is eliminated. This results in very conservative populations, in which the outcome of social transmission is, paradoxically, to eliminate almost all social transmission $[11,13,14]$. An intuitive justification for this result is that conservative individuals, for the very reason they are conservative, change more rarely than open individuals. Transitions from open to conservative thus occur more often than transitions from conservative to open. We will see in Sect. 3 that the drive toward conservatism can be overcome when the interactions of multiple cultural traits are considered, although it remains a powerful influence on cultural dynamics [10]. 


\subsection{Persuasiveness}

We define persuasiveness, $q$, as the probability that an individual causes another to change in a social interaction. We can show that cultural transmission favors high persuasiveness with the same reasoning leading to the conclusion that it favors low openness. In a social interaction between individuals $i$ and $j$ such that $i$ is more persuasive than $j, q_{i}>q_{j}$, we expect, on average, that $q_{i}$ decreases and $q_{j}$ increases:

$$
E\left[\Delta q_{i}\right]<0 \quad E\left[\Delta q_{j}\right]>0
$$

Since $i$ is more persuasive, $j$ is expected to change more:

$$
\left|E\left[\Delta q_{i}\right]\right|<\left|E\left[\Delta q_{j}\right]\right|
$$

It follows that the average persuasiveness is expected to increase:

$$
E\left[\frac{1}{2}\left(q_{i}+q_{j}\right)\right]=\frac{1}{2}\left(E\left[\Delta q_{i}\right]+E\left[\Delta q_{j}\right]\right)>0
$$

This dynamics has been studied in detail in previous work [11, 13, 14] and will be discussed further in Sect. 3.

\subsection{Social Networks: Whom to Listen to}

Social networks are widely recognized as important determinants of cultural dynamics, but it is equally important to study how cultural dynamics determines social networks (see, e.g., [15]). An individual's social network influences the transmission of other cultural traits, and thus cultural traits that modify individual's social networks are regulatory traits according to our definition. By analogy with the reasoning we made for openness, we expect cultural evolution to favor individuals who accept only a few others as cultural models. The reason is that such individuals have a smaller chance of changing than individuals who are willing to copy from many. We thus expect transitions from large to small social networks to occur more often than transitions in the reverse direction.

Let us assume that individual $i$ is susceptible of acquiring cultural traits only from $n_{i}$ cultural models, chosen at random or according to some rule. If $i$ copies one of her models, we assume for mathematical simplicity that she replaces her set of models with the model's set. ${ }^{1}$ We ask how the average of $n_{i}$ over the

\footnotetext{
${ }^{1}$ We have verified in computer simulations that this assumption is not crucial for our general argument, as long as $i$ 's set of models becomes more similar to that of her model as a result of the interaction.
} 
whole population changes during cultural evolution. In an interaction between two individuals $i$ and $j$ the probability that $i$ adopts $j$ 's set of models is the probability that $j$ is in $i$ 's set of models, i.e., $n_{i} / n$, where $n$ is the population size. If $i$ adopts $j$ 's models, $n_{i}$ becomes equal to $n_{j}$, hence the expected change in $n_{i}$ is

$$
E\left[\Delta n_{i}\right]=a \frac{n_{i}}{n}\left(n_{j}-n_{i}\right)
$$

where $a$ is the probability of learning from a cultural model in an interaction. Similarly, the expected change in $n_{j}$ is:

$$
E\left[\Delta n_{j}\right]=a \frac{n_{j}}{n}\left(n_{i}-n_{j}\right)
$$

The average expected change is thus

$$
E\left[\frac{1}{2}\left(\Delta n_{i}+\Delta n_{j}\right)\right]=\frac{1}{2} E\left[\Delta n_{i}\right]+\frac{1}{2} E\left[\Delta n_{j}\right]=-\frac{a}{2 n}\left(n_{j}-n_{i}\right)^{2}<0
$$

where we have used Eqs. (7) and (8) and noted that the term $n_{j}-n_{i}$ appears with opposite signs in the two equations. Hence the result of the interaction is to reduce the average number of cultural models, favoring small social networks.

\subsection{Social Networks: Whom to Talk to}

The results above show that cultural evolution tends to limit individuals' opportunities to be influenced by others. Conversely, it tends to increase individuals' opportunities to influence others. In addition to favoring high individual persuasiveness (Sect. 2.2), cultural evolution is also expected to favor individuals who try to influence a large number of others. The reasoning is analogous to the one in the previous section.

Let us assume that individual $i$ tries to convince of her own ideas only $m_{i}$ other individuals, which we may call $i$ 's "target set." If $i$ is copied by one of these individuals, the latter adopts $i$ 's target set as her own. In an interaction between individuals $i$ and $j$ the probability that $i$ adopts $j$ 's target set is the probability that $i$ is in $j$ 's target set, i.e., $m_{j} / n$, and the expected change in $m_{i}$ is

$$
E\left[\Delta m_{i}\right]=\frac{m_{j}}{n}\left(m_{j}-m_{i}\right)
$$

Similarly, the expected change in $m_{j}$ is:

$$
E\left[\Delta m_{j}\right]=\frac{m_{i}}{n}\left(m_{i}-m_{j}\right)
$$


The average expected change is thus

$$
E\left[\frac{1}{2}\left(\Delta m_{i}+\Delta m_{j}\right)\right]=\frac{1}{2 n}\left(m_{j}-m_{i}\right)^{2}>0
$$

which is obtained similarly to Eq. (9). This result shows that cultural evolution tends to produce individuals (or, generalizing, organizations such as religions or political parties) who, everything else being equal, try to influence as many others as possible, rather than limiting the set of potential cultural targets.

\section{The Far-Reaching Consequences of Regulatory Traits}

The models above show that cultural evolution can be profoundly shaped by the additive effects of repeated social interactions and that very simple assumptions can generate surprising results. We show below that, although we have so far considered rather idealized models, our results may shed light on actual cultural phenomena.

\subsection{In-Group Bias}

We saw in Sect.2.3 that we expect cultural evolution to reduce the number of individuals' cultural models (irrespective of whether such reduction improves, say, the individuals' well-being or genetic fitness). This result directly bears on actual social networks once we take into account that, in reality, the set of one's cultural models is not an arbitrary list of individuals, but is itself based on various cues such as social class, ethnicity, and gender.

Consider, for example, a population subdivided into a number of recognizable groups, and in which two rules for determining whom to copy exist: "copy everyone," and "copy only individuals from my own group." We expect the second rule to spread because it results in a smaller set of cultural models. Indeed, let $b_{i}$ be the number of individuals with in-group bias in group $i, g_{i}$ the size of group $i$, and $a$ the probability of learning from a cultural model. The expected number of individuals in group $i$ that acquire the in-group bias in each time step is

$$
a\left(g_{i}-b_{i}\right) \sum_{i} b_{i}
$$

where the last two factors are the probability that an unbiased individual in group $i$ (of which there are $g_{i}-b_{i}$ ) meets a biased individual from any group (of which there are $\sum_{i} b_{i}$ ). The expected number of group $i$ individuals who lose the in-group bias is

$$
a b_{i}\left(g_{i}-b_{i}\right)
$$


because the bias can be lost only copying an unbiased individual from one's own group, as individuals with the bias do not copy those from other groups. The expected change in the number of biased individuals in group $i$ is the difference of the two expressions above:

$$
E\left[\Delta b_{i}\right]=a\left(g_{i}-b_{i}\right) \sum_{i} b_{i}-a b_{i}\left(g_{i}-b_{i}\right)=a\left(g_{i}-b_{i}\right) \sum_{j \neq i} b_{j}
$$

which is always positive as long as $b_{i}<g_{i}$ (not everyone in group $i$ is biased) and there is at least one biased individual outside of group $i$. Thus the number of biased individuals is expected to increase until everyone is biased. While many mechanisms have been suggested to contribute to in-group bias [16] and other model-based biases $[17,18]$, it seems plausible that regulatory traits play a role in the origin of maintenance of such biases.

\subsection{Openness and Conservatism}

People, in real life, do not indiscriminately reject cultural information, and human populations do not become completely conservative, as predicted by the model described in Sect. 2.1. To investigate the circumstances under which a population can remain open to cultural influences, despite a tendency of cultural evolution to favor conservatism, we studied a more realistic model in which many cultural traits coevolve [10].

We modeled individuals as having both multiple cultural traits and preferences, i.e. positive or negative attitudes towards those traits. Preferences are themselves cultural traits that can be copied in social interactions. We assumed that the probability that an individual copies a potential cultural model is an increasing function of the individual's preference for the model's traits. Thus the probability to copy is highest when a model possesses many traits for which the observer has high preference. Low preferences make an individual conservative and should thus be favored by cultural evolution. Another force, however, promotes openness rather than conservatism. In fact, individuals who are too conservative fail to acquire cultural traits from others, and therefore cannot be copied since they don't display anything that observers can evaluate. Such an incentive to acquire traits is a form of the general incentive to persuade others described in Sect.2.2. Our simulations elucidated the interplay between these forces, showing that an open population can be maintained when there are many cultural traits and/or when the efficiency of cultural transmission is low (the latter regulates how many traits and preferences can be acquired in a single social interaction). Figure 1a, as an example of the latter effect, shows how average openness varies in time in two populations in which ten cultural traits are present, but that differ in $p_{\text {adopt }}$, i.e. the efficiency of transmission (for the effect related to the increase of the number of cultural traits, see [10]). 
a

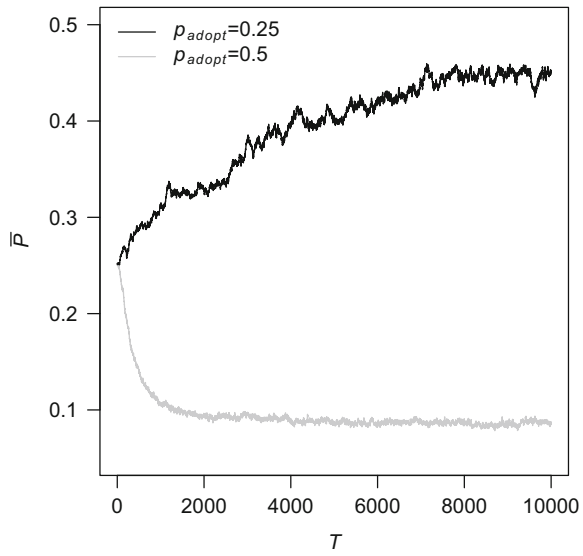

b

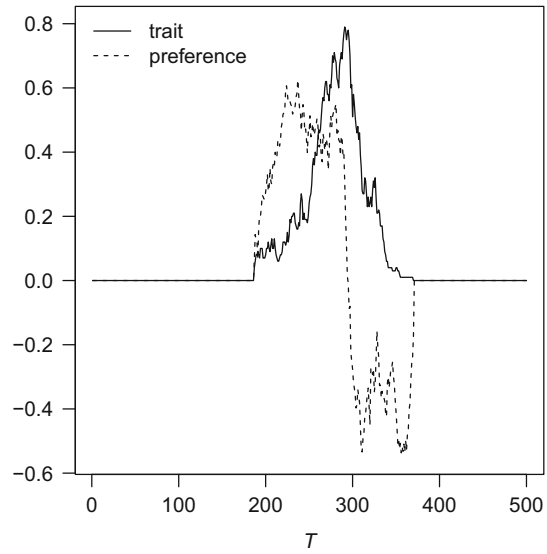

Fig. 1 (a) Average value of $P$ (openness, $X$ axis) through time ( $Y$ axis) in a population in which ten cultural traits are present, for an intermediate rate of transmission (black line: $p_{\text {adopt }}=0.25$ ) and for a higher rate of transmission (gray line: $p_{\text {adopt }}=0.5$ ). (b) An example of "fashion cycle." The solid line shows the frequency of the trait in the population through time, and the dashed line the average value of the preference associated with the trait. The graphs are redrawn from simulations detailed in [10] and [12], respectively

The increase of the number of cultural traits and the decrease of the efficiency of transmission have indeed an analogous effect on individual development: they both increase the number of interactions that an individual needs in order to acquire a substantial part of her culture. Successful cultural models are individuals who have many traits (so that they can influence others) and low preferences (so that they change rarely and thus repeatedly expose others to a stable set of traits). This combination can only be achieved by balancing openness and conservatism, remaining open during the first part of one's life, in order to learn as much culture as possible, and then become conservative to promote the spread of such culture $[10,12]$. If the number of cultural traits is large and/or the efficiency of cultural transmission is low, an individual needs to spend a good part of her life acquiring culture, and thus must remain open for a relatively long time. Such relatively open individuals will be better cultural models than conservative individuals who have not acquired much culture. Their traits will thus spread in the population, including the relatively high preferences that make them open. Thus a population in which individuals need a good part of their life to acquire enough culture to be good models is predicted to remain relatively open. This picture fits well with empirical data showing that individuals become more conservative with age [19], but that individuals with higher education-in our model individuals with many cultural traits—remain open to new information into adulthood and old age [20]. 


\subsection{Fashion Cycles}

In a later study [12], we used the trait-preference model to study the fate of cultural innovations. Rather than limiting culture to a fixed set of traits, we allowed individuals to occasionally introduce new cultural traits. Individual preferences for new traits were randomly set at the moment of trait introduction. Thus it may happen, by chance, that a currently influential individual (someone possessing many traits others prefer) has a high preference for a new trait. Such a high preference can then spread as the individual is copied often by others, which in turn drives the spread of the new trait because, when the preference is common, individuals with the trait are better cultural models than individuals without the trait. As the trait becomes common, however the situation changes. Individuals with a low preference for a common trait gain an advantage in transmitting their traits, because they are more conservative than others, as explained in Sect.2.1. This causes the low preference to spread, which in turn leads the population to abandon the trait, as possessing it is now a disadvantage. Figure $1 \mathrm{~b}$ shows the complete cycle just described.

This dynamics offers a plausible and parsimonious explanation of fashions and fads, according to which rises and fall in trait popularity are a universal emergent property of cultural evolution, hinging on regulatory traits dynamics. Well-known examples of fashions and fads include clothing styles [21] and the popularity of pop records [22]. Further data indicate that fashion phenomena are present in all times and cultures. Symbolic features of Polynesian canoes such as paintings on paddles, for example, change more rapidly than functional features such as the shape of the hull [23], and analysis of decorative motifs in Neolithic pottery is consistent with the idea that individuals, or households, copied each other's motifs through time [24].

Our model explains two quantitative features of empirical data (compare Figs. 2 and 3). The first (Fig. 2a) is the power-law, or log-normal, distribution of frequency of cultural traits [25], meaning that only very few cultural traits become very common while the vast majority remains rare. This effect has been observed in several cultural domains, including, among others, first names, scientific citations, books translations, popularity of dog breeds (see [22, 25]). The second (Fig. 2b) is the finding that cultural traits that increase rapidly in popularity are also abandoned quickly, while slow increases in popularity correlate with slow decreases (shown for first names in the USA and France [26], and for dog breeds in the USA [12]).

The model accounts for these findings better than two common alternative views of fashion. The first is generally called neutral model of cultural evolution and simply assumes that individuals copy each other randomly [25]. This model has convincingly been shown to reproduce the long-tailed distributions of frequency of cultural traits, but fails in reproducing the correlation between the rates of increase and the rates of decrease of popularity. If individuals copy each other randomly, in fact, increases and decreases of popularity of a trait depend exclusively on the frequency of the trait at each time step, while the correlation requires some sort of "memory" (in our model provided by the coevolution of preferences and traits). 
a

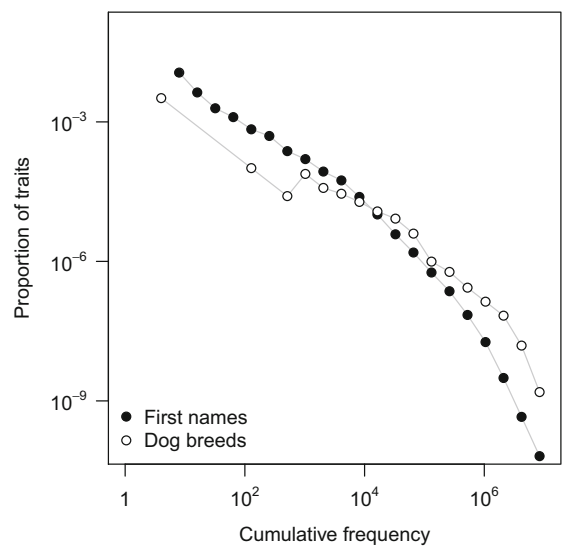

b

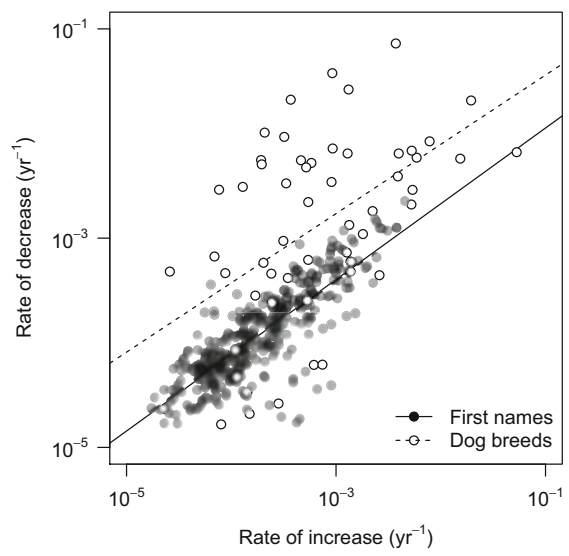

Fig. 2 Empirical findings on fashion cycles. (a) Distribution of frequency of cultural traits. (b) Rates of increase and rates of decrease of popularity of cultural traits. For both panels, closed circles are first names in USA 1880-2006, and open circles are dog breeds registered with the American Kennel Club, 1926-2005 (courtesy of Herzog)

a

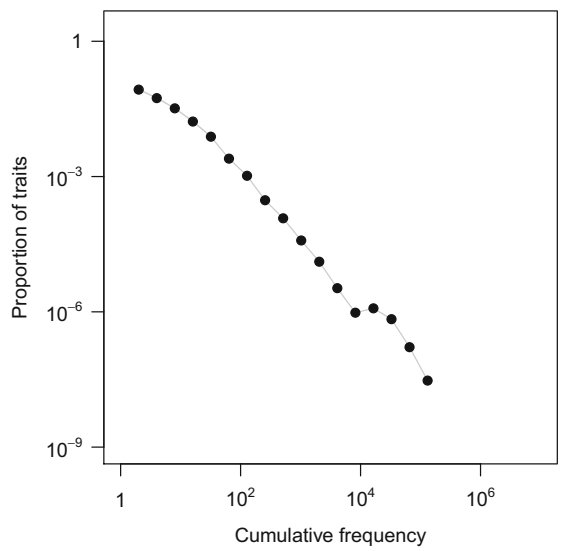

b

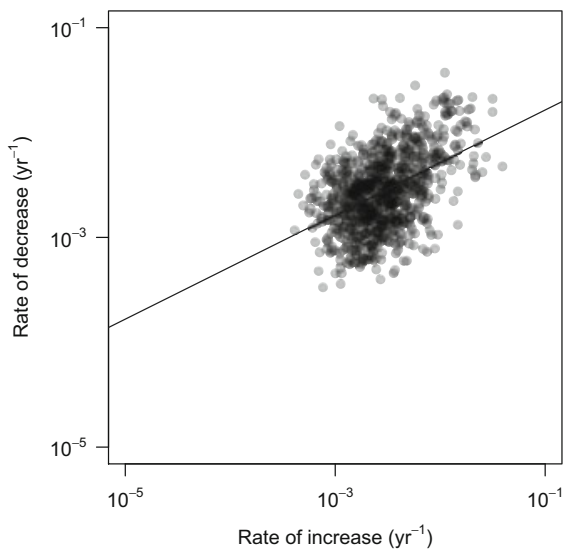

Fig. 3 Simulations results on fashion cycles. (a) Distribution of frequency of cultural traits. (b) Rates of increase and rates of decrease of popularity of cultural traits. Simulated time steps have been converted to years assuming an average lifetime of 70 years. The graphs are redrawn from simulations detailed in [12]

The second common view considers fashion cycles a direct result of social stratification [27]. According to this view, a cycle starts when individuals of low social status copy individuals of perceived high status. When a trait become common, however, high-status individuals abandon it to differentiate themselves 
from low-status individuals, and, as a consequence, low-status individuals abandon it too. This status model, contrarily to the neutral model, generates correlations between rates of increase and rates of decrease of popularity, but not long-tailed frequency distributions. Indeed, as soon as a cultural trait become common, highstatus individuals abandon it, triggering abandonment from low-status individuals. While the status model may describe brief fads, it seems unable to account for cultural traits that exhibit long-lasting popularity, for example English names such as Mary and John.

\section{Discussion}

In this chapter we extended our previous works to present a general argument about the importance of regulatory traits in cultural evolution. Our results show that regulatory traits may have a potent, and perhaps surprising, impact on cultural dynamics. Cultural evolution, in other words, can generate its own rules [13] in absence of extra-cultural driving forces, such as natural selection or memory mechanisms. This does not mean that these factors are unimportant, but studying pure cultural forces with mathematical and simulation models may help us to isolate and elucidate the effects of such forces.

A more general question is how the existence of regulatory traits impacts on the analogy between cultural and genetic evolution and, specifically, on the possibility to model cultural dynamics using models inspired by evolutionary biology. Note that "regulatory genes" are not analogous to cultural regulatory traits. Regulatory genes activate or inhibit other genes [28]; they do not alter how genetic material is transmitted. A genuine example of genetic regulatory trait, in the sense we use the term, is genes that determine the mode of reproduction (sexual vs. asexual), which are found in some species $[29,30]$.

Genetic regulatory traits, however, appear rare in genetic evolution, as witnessed by the remarkable success of population genetic models that employ immutable rules of genetic transmission [31-33]. By contrast, regulatory traits seem to be widespread in cultural evolution. Why is it so? Information transfer (or modes of transmission [7]) is highly constrained in genetic evolution. Although more flexible modalities of gene transfer exist [34,35], genes typically propagate to offspring from just two (sexual reproduction of chromosomal DNA) or one parent (asexual reproduction or sexual reproduction of mitochondrial DNA). Cultural information instead can be transmitted in many different ways and, potentially, from any individual to any other individual, which creates the opportunity to regulate the flow of information in a more fine-grained and context-dependent way. Claidière and André [7] recently proposed that the instability of modes of transmission in culture is one of the major differences between cultural and genetic evolution. Our results suggest, indeed, that cultural forces can favor different kinds of information flow. We believe that the concept of regulatory traits can be important to identify and understand when and how this may happen. 
Models of cultural evolution inspired by evolutionary biology have been criticized for their excessive simplicity, which has sometimes lead to a rejection of modeling altogether, especially within anthropology [36]. This is certainly not our conclusion. The possibility of applying models already developed in other disciplines to the study of culture is certainly positive. Modeling efforts, however, should progress toward a richer characterization of cultural dynamics. We hope to have shown that a complete theory of cultural evolution cannot ignore that cultural transmission can be more flexible than genetic transmission.

Acknowledgments Work supported by the Uniquely Human project funded by the Swedish Research Council.

\section{References}

1. Boyd, R., Richerson, P.J.: Culture and the Evolutionary Process. University of Chicago Press, Chicago (1985)

2. Cavalli-Sforza, L.L., Feldman, M.W.: Cultural Transmission and Evolution: A Quantitative Approach. Princeton University Press, Princeton (1981)

3. Lewontin, R.C.: The units of selection. Annu. Rev. Ecolo. Syst. 1, 1-18 (1970)

4. Mesoudi, A.: Cultural Evolution. How Darwininan Evolutionary Theory Can Explain Human Culture and Synthesize the Social Sciences. University of Chicago Press, Chicago (2011)

5. Sperber, D., Claidière, N.: Why modeling cultural evolution is still such a challenge. Biol. Theor. 1(1), 20-22 (2006)

6. Strimling, P., Enquist, M., Eriksson, K.: Repeated learning makes cultural evolution unique. Proc. Natl. Acad. Sci. 106(33), 13870-13874 (2009)

7. Claidière, N., André, J.-B.: The transmission of genes and culture: a questionable analogy. Evol. Biol. 39(4), 12-24 (2012)

8. Acerbi, A., Parisi, D.: Cultural transmission between and within generations. J. Artif. Soc. Soc. Simulat. 9(1), 9 (2006)

9. Henrich, J., McElreath, R.: The evolution of cultural evolution. Evol. Anthropol. 12, 123-135 (2003)

10. Acerbi, A., Enquist, M., Ghirlanda, S.: Cultural evolution and individual development of openness and conservatism. Proc. Natl. Acad. Sci. 106(45), 18931-18935 (2009)

11. Ghirlanda, S., Acerbi, A., Enquist, M., Nakamaru, M.: The sometimes evitable route to conservatism and persuasiveness. Curr. Anthropol. 51(2), 271-272 (2010)

12. Acerbi, A., Ghirlanda, S., Enquist, M.: The logic of fashion cycles. PLoS One 7(3), e32541 (2012)

13. Ghirlanda, S., Enquist, M., Nakamaru, M.: Cultural evolution develops its own rules. The rise of conservatism and persuasion. Curr. Anthropol. 47(6), 1027-1034 (2006)

14. Xue, J.Z., Costopolous, A.: The evitable route to zelaotry. Curr. Anthropol. 51(2), 269-270 (2010)

15. Centola, D., Gonzalez Avella, J.C., Eguiluz, V.M., San Miguel, M.: Homophily, cultural drift, and the co-evolution of cultural groups. J. Conflict Resolut. 51(6), 905-929 (2007)

16. Hewstone, M., Rubin, M., Willis, H.: Intergroup bias. Annu. Rev. Psychol. 53, 575-604 (2002)

17. Henrich, J.: Cultural transmission and the diffusion of innovations: adoption dynamics indicate that biased cultural transmission is the predominate force in behavioral change. Am. Anthropol. 103(4), 992-1013 (2001)

18. Richerson, P.J., Boyd, R.: Not by Genes Alone: How Culture Transformed Human Evolution. The University of Chicago Press, Chicago (2005) 
19. Caspi, A., Roberts, B.R., Shiner, R.L.: Personality development: stability and change. Annu. Rev. Psychol. 56, 453-484 (2005)

20. Costa, P.T.J., McCrae, R.R.: Comprehensive Handbook of Personality and Psychopathology. Wiley, Hoboken (2006)

21. Belleau, B.D.: Cyclical fashion movement: women's day dresses: 1860-1980. Cloth. Text. Res. J. 5(2), 15-20 (1987)

22. Bentley, R.A., Lipo, C.P., Herzog, H.A., Hahn, M.: Regular rates of popular culture change reflect random copying. Evol. Hum. Behav. 28, 151-158 (2007)

23. Rogers, D.S., Ehrlich, P.R.: Natural selection and cultural rates of change. Proc. Natl. Acad. Sci. 105(9), 3416-3420 (2008)

24. Bentley, R.A., Shennan, S.J.: Cultural transmission and stochastic network growth. Am. Antiquity 68(3), 458-485 (2003)

25. Bentley, R.A., Hahn, M., Shennan, S.J.: Random drift and culture change. Proc. Roy. Soc. Lond. B 271, 1443-1450 (2004)

26. Berger, J., Le Mens, G.: How adoption spread affects the abandonment of cultural tastes. Proc. Natl. Acad. Sci. 106(20), 8146-8150 (2009)

27. Simmel, G.: Fashion. Int. Q. 10, 130-155 (1904)

28. West-Eberhard, M.J.: Developmental Plasticity and Evolution. Oxford University Press, New York (2003)

29. Stelzer, C.-P., Schmidt, J., Wiedlroither, A., Riss, S.: Loss of sexual reproduction and dwarfing in a small metazoan. PLoS One 5(9), e12854 (2010)

30. Sandrock, C., Vorburger, C.: Single-locus recessive inheritance of asexual reproduction in a parasitoid wasp. Curr. Biol. 27, 433-437 (2011)

31. Crow, J.F., Kimura, M.: Evolution in sexual and asexual populations. Am. Nat. 99, 439-450 (1965)

32. Maynard Smith, J.: The Evolution of Sex. Cambridge University Press, Cambridge (1978)

33. Futuyma, D.J.: Evolutionary Biology. Sinauer, Saunderland (1998)

34. Gogarten, J.P., Townsend, J.P.: Horizontal gene transfer, genome innovation and evolution. Nat. Rev. Microbiol. 3, 679-687 (2005)

35. Keeling, P.J., Palmer, J.D.: Horizontal gene transfer in eukaryotic evolution. Nat. Rev. Genet. 9, 605-618 (2008)

36. Ingold, T.: The trobule with 'evolutionary biology'. Anthropol. Today 23(2), 13-17 (2007) 